\title{
Energieeffizienznetzwerke - beschleunigte Emissionsminderungen in der mittelständischen Wirtschaft
}

\author{
Eberhard Jochem • Michael Mai • Volker Ott
}

Online publiziert: 11. März 2010

(C) The Author(s) 2010. Dieser Artikel ist auf Springerlink.com mit Open Access verfügbar

Zusammenfassung Die Verminderung der energiebedingten $\mathrm{CO}_{2}$-Emissionen in der mittelständischen Wirtschaft durch effizientere Nutzung von Energie ist eine der rentabelsten Optionen. Dennoch werden diese Chancen wegen vielfältiger Hemmnisse und Marktversagen kaum realisiert. Hierbei spielen hohe Transaktionskosten und Entscheidungsroutinen bei Investitionen und beim Einkauf der Betriebe eine erhebliche Rolle. Ein in der Schweiz entwickeltes Netzwerkkonzept mit Eingangsberatung jedes teilnehmenden Betriebes, Zielsetzungen für das gesamte Netzwerk, regelmäßigen moderierten Treffen der Energiebeauftragten zum Erfahrungsaustausch sowie einem jährlichem Monitoring überwindet viele dieser Hemmnisse und führt zu einer Verdopplung des energietechnischen Fortschrittes relativ zum Effizienzfortschritt der Industrie insgesamt. Dieses Netzwerkkonzept kann weitestgehend von der Wirtschaft selbst durchgeführt werden. Die jährliche durchschnittliche Energiekostenersparnis ist rd. $100.000 €$ je Betrieb und Jahr und die $\mathrm{CO}_{2}$-Emissionsminderung rd. 500 t $\mathrm{CO}_{2}$. Bei einem Gesamtpotential von rd. 700 Netzwerken wären für 2020 Emissionsminderungen bis $\mathrm{zu} 10 \mathrm{Mio}$. $\mathrm{CO}_{2}$ möglich. Ein Netzwerk-Managementsystem erlaubt einen Mindeststandard zum Aufbau und Betrieb derartiger Netzwerke für Beratende Ingenieure und Moderatoren.

E. Jochem $(\varangle)$

CEPE, ETH Zürich, Zürichbergstr. 18, 8082 Zürich, Schweiz

e-mail: ejochem@ethz.ch

\section{Mai}

IREES, Schönfeldstr. 8, 76131 Karlsruhe, Deutschland

e-mail:m.mai@irees.de

V. Ott

Fraunhofer-ISI, Breslauerstr. 48, 79139 Karlsruhe, Deutschland

e-mail: v.ott@isi.fraunhofer.de

\section{Energy efficiency networks-accelerated mitigation of $\mathrm{CO}_{2}$ emissions in medium sized companies}

\begin{abstract}
The reduction of energy-related $\mathrm{CO}_{2}$ emissions by more efficient enery use in medium sized companies is one of the most profitable options. However, these opportunities are scarcely realised because of many obstacles and market failures. High transaction cost and decision routines play an important role for decision making and procurement of the companies. A network concept being developed in Switzerland and covering an initial consulting of each participating company, targets for the network, regular and moderated meetings of the energy managers for mutual exchange of experiences and a yearly monitoring alleviates many of these obstacles. It doubles the energy efficiency progress relative to the average progress of industry. This network concept can be fully realised by industry itself. Average energy savings per site and year are 100,000 $€$ and average $\mathrm{CO}_{2}$ emission reduction about 500 t $\mathrm{CO}_{2}$ per year and site. Assuming a maximum potential of 700 Networks, additional emission reductions of some $10 \mathrm{Mio}$. $\mathrm{CO}_{2}$ seem to be possible in Germany by 2020. A network management system for consulting engineers and moderators allows a minimum performance standard of how to start and operate those efficiency networks.
\end{abstract}

\section{Gebremste Nutzung rentabler Energieeffizienz-Optionen}

Die $\mathrm{CO}_{2}$-Emissionen der deutschen Wirtschaft betragen derzeit rund 350 Mio. t, einschließlich der indirekten Emissionen bei der Stromerzeugung. Hier steckt ein großes 
Minderungspotential durch einen effizienteren Umgang mit Energie und durch Substitution der derzeit verwendeten Energieträger. Die durchschnittliche Steigerung der Energieeffizienz lag in der deutschen Wirtschaft in den letzten zehn Jahren bei gut einem Prozent pro Jahr. Dieser ,,autonome" energiesparende Fortschritt spiegelt nach den Erkenntnissen beratender Ingenieure bei weitem nicht die rentablen Energieeffizienzmöglichkeiten wider, die Betriebe der mittelständischen Wirtschaft realisieren könnten.

Diese Erkenntnis ist nicht neu und wird traditionell mit vorliegenden Hemmnissen und Marktversagen erklärt (DeGroot 2001). Auf einzelne Hemmnisse - aber nicht auf die Gesamtheit der Hemmnisse - gehen energiepolitische Maßnahmen ein wie z.B. finanzielle Förderung von Beratungen, Investitionszuschüsse oder technische Standards bei massengefertigten Produkten wie z.B. Elektromotoren. Dieser Artikel erläutert daher zunächst kurz die zentralen Hemmnisse, warum in der mittelständischen Wirtschaft heute rentable Energieeffizienz-Maßnahmen nur zögerlich umgesetzt werden und schildert dann ein neues, in den letzten Jahren in Deutschland erprobtes Instrument, die Energieeffizienzund Klimaschutz-Netzwerke, mit seinen Ergebnissen zur beschleunigten Energiekostensenkung und beschleunigten $\mathrm{CO}_{2}$-Minderung.

\subsection{Wesentliche Hemmnisse zur Realisierung von Energieeffizienz-Potentialen in der mittelständischen Wirtschaft}

Beratende Ingenieure berichten immer wieder von der Tatsache, dass es zahlreiche rentable Energieeinspar- und damit auch rentable $\mathrm{CO}_{2}$-Minderungspotenziale gibt, d.h. Investitionen oder organisatorische Maßnahmen an Maschinen, Anlagen und Gebäuden, die von den Betrieben nicht realisiert werden. Die Gründe dafür sind zahlreich (Sorrell et al. 2000; DeCanio 1998, 1993; DeGroot 2001):

- Das Interesse an Energieeffizienzverbesserung ist häufig deshalb so gering, weil Energie nur eine Hilfsfunktion hat: Die verlässliche Verfügbarkeit von Strom und Wärme ist für die Produktion wichtig, nicht aber deren möglichst effizienter Einsatz, zumal die Energiekostenanteile an den gesamten Produktionskosten mit durchschnittlich 1,5 bis $3 \%$ in der Industrie sehr gering sind (Ausnahme: die Grundstoffindustrie).

- Zudem fluktuieren Energiepreise erfahrungsgemäß, während man davon ausgeht, dass in Zukunft die Löhne auf alle Fälle steigen, d.h., die Erhöhung der Arbeitsproduktivität hat stets hohe Priorität.

- Die Such- und Entscheidungskosten für EnergieeffizienzInvestitionen (Transaktionskosten) sind - gemessen an den zusätzlich einzusparenden Energiekosten - für viele Investoren oder Geschäftsleitungen relativ hoch (Ostertag 2003); derartige Entscheidungen kommen zu selten vor, eine professionelle Beratung fehlt oder wird nicht wahrgenommen, sei es aus Unkenntnis oder infolge schlechter Erfahrungen.

- Externe Energieberatung wird auch deshalb nicht in Anspruch genommen, weil man den Nettonutzen nicht kennt oder der technische Betriebsleiter sein Gesicht nicht verlieren will.

- Etwa 80\% der Unternehmen in Deutschland entscheiden ihre Investitionen nach der Amortisationszeit, d.h. einem reinen Risikomaß. Da die Energieeffizienz-Investitionen aber meist Lebensdauern von 10 Jahren und mehr haben (bei Gebäudeteilen bis zu 30 Jahren und mehr), wird systematisch mit diesem Entscheidungskriterium die energie-ineffiziente Option bevorzugt.

- Das gleiche gilt für die Minimierung der Investitionen, wobei der Einkauf der Betriebe zu wenig Spezifikationen für Energieeffizienz erhält und nach den Lebenszykluskosten nicht frägt. Dieses Einkaufsverhalten zwingt die Hersteller von Anlagen und Maschinen in sehr vielen Fällen, über den reinen Preiskampf der Investition energetisch zweitklassigen Lösungen anzubieten.

Diese Hemmnisse sind im wesentlichen auf der ökonomischen Ebene angesiedelt. Im Laufe des Projektes traten mehrere dieser Hemmnisse, insbesondere die beiden letztgenannten, in den Fokus der Beobachtungen.

\subsection{Sozialpsychologische Aspekte bei der Realisierung von Energieeffizienz-Maßnahmen}

Diese beobachteten Hemmnisse und entsprechende energiepolitische Instrumente wie finanzielle Anreize, Ökosteuer, technische Standards, Informationsbroschüren sowie die gängige Beratungstätigkeit tragen den sozialpsychologischen Mechanismen innerhalb und zwischen den Unternehmen der Wirtschaft zu wenig Rechnung. Fast unbeachtet bleiben die möglichen Wirkungen von Kommunikation zwischen den Unternehmen oder die Kommunikation innerhalb der Unternehmen für erfolgreiche Projekte. Dabei kann die durch Erfahrungsaustausch, gegenseitige Bestätigung und Anerkennung oder die Steigerung des Sozialprestiges von Energiebeauftragten oder Betriebsleitern ausgelöste Motivation Lerneffekte mit weit reichenden Auswirkungen auf betrieblichen Nutzen und Umweltvorteilen auslösen (Stern 1992; Jochem et al. 2000; Schmid 2003).

Die zentralen Erkenntnisse aus der Auswertung Lernender Netzwerke gehen alle davon aus, dass ein durch persönliche Kontakte getragener Erfahrungsaustausch zu Synergieeffekten durch gemeinsames Lernen führt. Die vertrauensvolle, unmittelbare Übernahme von Erfahrungen reduziert die Transaktionskosten in Netzwerken substantiell (Scott 2000; Jochem et al. 2009).

Die Diffusion von Innovationen erfolgt nach neueren Erkenntnissen nicht allein über lineare Informationsprozesse von einer Quelle über Multiplikatoren zu Empfängern. 
Vielmehr sind es interaktive Vernetzungen zwischen Marktakteuren, zwischen denen Erfahrungen ausgetauscht werden, zwischen denen gemeinsames Lernen und gemeinsame Entwicklungen möglich sind (Aijzen 1991). Netzwerke dieser Art sind institutionalisierte Formen der Kooperation und Kommunikation (Jansen 1999). Ein „Milieu“ des Austausches von Ideen, Problemen und Lösungen begünstigt die Diffusion von Innovationen auch im EnergieeffizienzBereich (Dosi 1988).

Nicht nur zwischen den Unternehmen und ihren Energieverantwortlichen spielen soziale Beziehungen eine Rolle, sondern auch innerhalb von Unternehmen. Einflussfaktoren für erfolgreiche Anstrengungen zur Verbesserung der Energieeffizienz sind auch die intrinsische Motivation von Unternehmensakteuren und Entscheidungsträgern, die Interaktion z.B. zwischen Energieverantwortlichen und Geschäftsleitung, interne Impulse von Schlüsselakteuren und deren Prestige und Überzeugungskraft sowie die Unternehmenskultur (InterSEE 1998; Schmid 2003). Für die Initiierung von Maßnahmen ist es auch wichtig, dass die relevanten Akteure sich die Umsetzung eines Projektes selbst zutrauen. Nicht selten scheuen die Produktionsingenieure Veränderungen an den Anlagen und Maschinen, um Risiken zur Produktqualität oder Produktionsstillstand zu vermeiden.

\section{Lernende örtliche Energieeffizienz-Netzwerke}

Diese Überlegungen und Beobachtungen führten in der Schweiz Mitte der 1980er Jahre zu einer Bildung örtlicher lernender Netzwerke, in denen jeweils etwa 15 bis 20 Betriebe sich in regelmäßigen Abständen zu einem moderierten Erfahrungsaustausch trafen (ModellSchweiz; Bürki 1999). Diese Treffen erweisen sich als sehr beschleunigend für den technischen Stand des Wissens der Beteiligten und zu etwa doppelt so schnellen Energieeffizienzfortschritten wie die Schweizer Industrie insgesamt (Kristof et al. 1999; Konersmann 2002). In Deutschland starteten dann die ersten derartigen Energieeffizienz-Netzwerke in einem ländlichen Raum (Hohenlohe) und einer Stadt (Ulm), beide gefördert vom Baden-Württembergischen Umweltministerium (Loistl u. Weissenbach 2007). In beiden Fällen wurden die positiven Ergebnisse der Schweiz wiederholt (Jochem et al. 2006; Jochem et al. 2007; Jochem u. Gruber 2007) und waren Anlass zu weiteren Gründungen derartiger Netzwerke in BadenWürttemberg und Bayern sowie erstmalig durch ein Energieversorgungsunternehmen, das in den letzten fünf Jahren in ganz Deutschland 12 Energieeffizienz-Netzwerke startete (Jochem et al. 2009; Wagner 2008).

Ziel eines lernenden EnergieEffizienz-Netzwerkes mit 10 bis 15 Unternehmen ist es, durch einen regelmäßigen, von einem Fachmann moderierten Erfahrungsaustausch die vielen rentablen Effizienz-Potentiale für jeden der Teilnehmer schneller und mit geringerem Aufwand für die Suche und Entscheidung einer energieeffizienten Lösung verfügbar zu machen. Nach den bisherigen Erfahrungen in der Schweiz und in Deutschland lässt sich bei den Teilnehmern derartiger Netzwerke die Verbesserung der Energieeffizienz und dadurch die Senkung der spezifischen Energiekosten um mindestens das Doppelte gegenüber dem industriellen Durchschnitt beschleunigen.

\subsection{Die Teilnehmer eines Energieeffizienz-Netzwerkes}

Zielgruppe der Netzwerke sind mittlere Betriebe bzw. Unternehmen, die Jahresenergiekosten von wenigstens $150.000 €$ bis $200.000 €$ aufweisen. Die Jahresenergiekosten eines Betriebes sollten allerdings auch nicht mehr als 50 Mio. $€$ betragen. Die maximal 15 Teilnehmer eines Netzwerkes kommen wegen der lokalen Orientierung meist aus verschiedenen Branchen, es sollten keine Unternehmen am selben Netzwerk teilnehmen, die als Wettbewerber den gleichen Kunden haben. Themenschwerpunkte der Netzwerke sind die Querschnittstechnologien wie z.B. Druckluft, Kälte, Wärmeerzeugung, Pumpen, Gebäudetechnik, Beleuchtung und Abwärmenutzung sowie Energiemanagement und Wirtschaftlichkeitsberechnungen. Entsprechend sollten hier die Betriebe eine breite Anwendung dieser Technologien aufweisen.

\subsection{Die drei Phasen des Netzwerk-Aufbaus und -Betriebs}

Die Initiierungsphase (vgl. Abb. 1) umfasst die Zeit von der Entscheidung der initiierenden Institution, ein lernendes Netzwerk aufzubauen bis zum ersten Netzwerk-Treffen der vertraglich eingebundenen Betriebe bzw. Unternehmen; diese Zeitspanne beträgt meist vier bis sechs Monate. Die initiierende Institution kann z.B: das Innovations-Referat einer IHK, ein regionales Wirtschaftsforum, ein Wirtschaftsförderer, eine Energie-Agentur, ein Amt einer Stadtverwaltung oder ein Energieversorgungsunternehmen sein. Eine ausführliche Beschreibung der Initiierungsphase mit Hilfen zur Unterstützung des Prozesses bietet ein in den letzten Jahren entwickeltes Netzwerk-Managementsystem (mit der Marke ${ }^{\circledR}$ LEEN, Local Energy Efficiency Network; Jochem et al. 2009).

Der Verlauf der Netzwerkfindung ist sehr abhängig von den vorhandenen persönlichen Kontakten mit Unternehmen sowie dem Ansehen und der Überzeugungskraft des Initiators bzw. der initiierenden Institution. Der Initiator sollte über möglichst breite Kontakte zu potentiell interessierten Betrieben am Ort oder in der Region sowie über kommunikative Fähigkeiten verfügen. Der Initiator sollte über ein Zeitbudget verfügen.

Die initiierende Institution muss nicht zwingend später der Netzwerkträger sein. Fallen initiierende Institution 
Abb. 1 Initiierung eines Energieeffizienz-Netzwerkes (Phase 0) und sein zeitlicher Ablauf mit Initialberatung und Zielsetzung (Phase 1) sowie moderierten Treffen und jährlichem Monitoring (Phase 2)

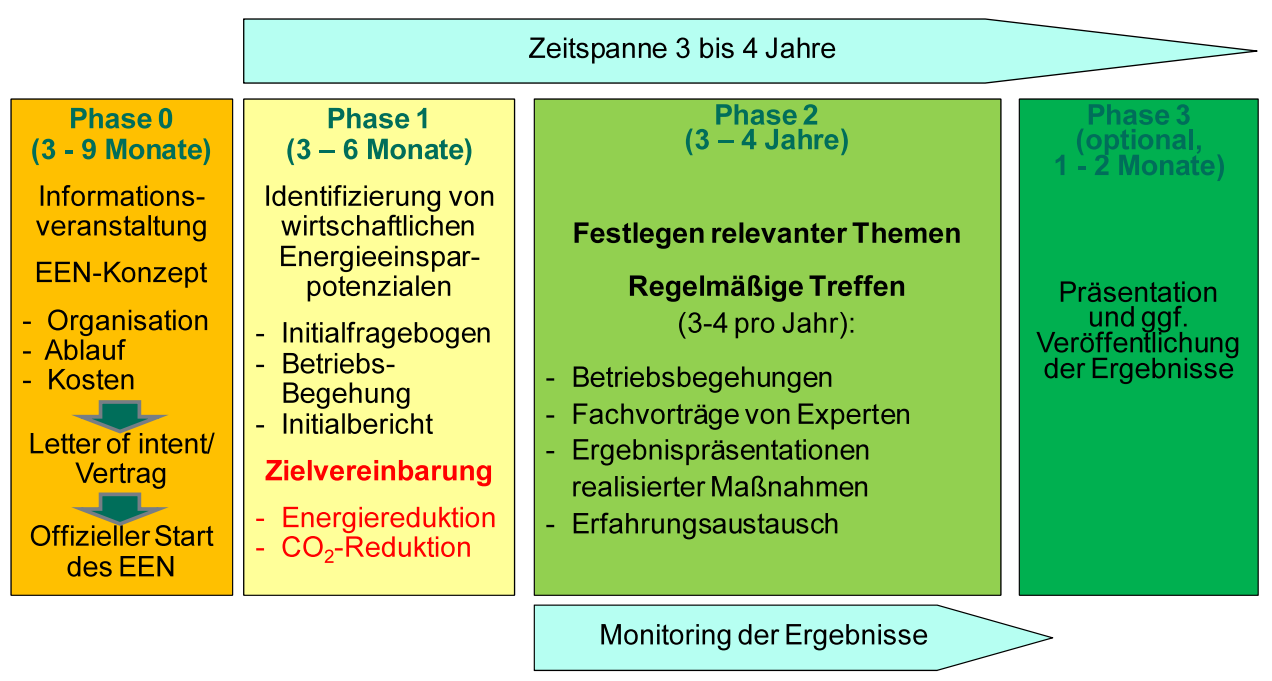

(z.B. örtliche IHK) und der Netzwerkträger auseinander, sollte der künftige Netzwerkträger (z.B. Wirtschaftsplattform, Energie-Agentur, Energieversorger) den zu gewinnenden Unternehmen möglichst früh bekannt sein (z.B. durch die Mitträgerschaft bei der Einladung zur ersten Informationsveranstaltung). Es ist wichtig, dass der künftige Netzwerkträger bei den beteiligten Unternehmen das Vertrauen für die Aufgabe der Ausschreibungen, der Vertragsgestaltung und der Organisation des Netzwerkes hat.

In der Phase 1 erfolgt für jeden Betrieb eine Erstberatung mit einem abschließenden Bericht, der auch der Geschäftsführung erläutert wird. Auf der Basis der Ergebnisse der Berichte aller Betriebe wird dann ein gemeinsames Ziel zur Effizienzverbesserung und zur Senkung der spezifischen $\mathrm{CO}_{2}$-Emissionen für das gesamte Netzwerk festgelegt, das in der Regel einen Zeithorizont von drei bis vier Jahren hat.

Nach Abschluss der Phase 1 mit der Zielfestlegung läuft dann die Arbeitsphase 2 mit meist jährlich vier Treffen zum Erfahrungsaustausch und einem jährlichen Monitoring für jeden Betrieb und für das Netzwerk als ganzes. Auf den vier Treffen, die immer in einem der beteiligten Betriebe stattfinden, werden jeweils ein bis zwei zuvor festgelegte Themen behandelt. Hierzu können auch externe Fachleute eingeladen werden. Mit der Dauer des Erfahrungsaustausches wächst das gegenseitige Vertrauen der Energiebeauftragten, und der Moderator kann den mehrjährigen Erfahrungsaustausch durch Auswahl interessanter Themen und Referenten aus dem Netzwerk selbst, einem anderen Netzwerk oder eines externen Fachmanns optimal steuern. Ein derartig professionell angelegter Erfahrungsaustausch von Energiebeauftragten senkt die Transaktionskosten in erheblichem Umfang. sie werden derzeit in einem Forschungsvorhaben erhoben.

Ziel des jährlichen Monitorings ist es, den einzelnen Netzwerkteilnehmern einen Einblick über die erreichte Energieeffizienzverbesserung und ihrer verminderten spe- zifischen $\mathrm{CO}_{2}$-Emissionen zu geben. Zudem wird die Frage beantwortet, inwieweit das Netzwerk insgesamt auf dem Zielpfad ist bzw. die gesetzten Ziele erreicht wurden. Längerfristig könnte das Monitoring auch dazu dienen, um z.B. - wie in der Schweiz - eine Befreiung des sich zu einem Ziel verpflichtenden Unternehmens von einer steuerlichen Belastung (z.B. Ökosteuer, $\mathrm{CO}_{2}$-Abgabe) zu erreichen.

\subsection{Kosten des Betriebes eines Netzwerkes und deren Finanzierung}

Die Kosten für die Netzwerkarbeit, d.h. die Initialberatung jedes einzelnen Teilnehmers, die Moderation der etwa viermal pro Jahr stattfindenden Treffen sowie für das jährliche Monitoring aller teilnehmenden Betriebe und das Projektmanagement, betragen für drei bis vier Jahre Netzwerkbetrieb im Durchschnitt etwa 60.000 bis $80.000 €$ pro Jahr für 10 Betriebe und etwa 85.000 bis $110.000 €$ pro Jahr für 15 Teilnehmer.

Diese Kosten werden in der Regel von den teilnehmenden Betrieben getragen. Manchmal beteiligen sich Sponsoren an den Kosten. 30 neue Energieeffizienz-Netzwerke werden derzeit vom Bundesumweltministerium gefördert, indem bis zu einem Drittel die Kosten für den Netzwerkbetrieb übernommen werden (www.30pilot-netzwerke.de).

\section{Ergebnisse}

Dieser Absatz fasst die Ergebnisse einer Analyse zusammen, die bei der Beobachtung von knapp 10 in Deutschland laufenden Energieeffizienz-Netzwerken gewonnen werden konnten. Dabei handelte es sich um rund 115 Betriebe der mittelständischen Wirtschaft.

Potentiale der Energiekostensenkung und der erneuerbaren Energien 
- Die in den Initialberatungen gefundenen rentablen Energieeffizienz-Potentiale betragen zwischen $4 \%$ (bei sehr energieintensiven Betrieben) und nicht selten bis über $20 \%$ (bei Betrieben der Konsum- und Investitionsgüterindustrie und der Dienstleistungssektoren) in den kommenden fünf bis acht Jahren. Die Potentiale sind sowohl organisatorische Maßnahmen, die sofort umgesetzt werden können (z.B. Vermeidung von Leckagen in Druckluftanlagen) als auch investive Maßnahmen, die im ReInvestitionszyklus in der Regel sehr hohe Rentabilitäten (meist mehr als 20\% interne Verzinsung) aufweisen.

- In den ersten beiden Jahren sind die organisatorischen Maßnahmen und Stromeffizienz-Investitionen am schnellsten umsetzbar, während die Effizienz-Investitionen in Abwärmenutzung oder effizientere Prozesswärme- und Kältenutzung einen Planungsvorlauf erfordern. Nach vier bis fünf Jahren Netzwerkbetrieb kommen verstärkt Investitionen in Kraft-Wärme-Kopplung (meist BHKWs), baulicher Wärmeschutz und Optimierung/Erneuerung der Produktionsanlagen in den Fokus.

- Wenn in den Betrieben pro Jahr etwa 100 Effizienzmaßnahmen realisiert wurden, so entstanden etwa 60 neue Ideen pro Jahr für weitere Effizienzmaßnahmen infolge genauerer Kenntnisse des jeweiligen Betriebes und infolge technischer Innovationen (z.B. Absorption brennbarer Inhaltsstoffe in Prozessabgasen, Leistungselektronik mit Netzrückspeisung von Bremsenergie).

- Der Einsatz der erneuerbaren Energien spielte bis 2007 praktisch keine Rolle, hat aber seit 2008 - wenn auch abgeschwächt durch den Erdölpreisverfall - bei den Betrieben eine größere Aufmerksamkeit bei den Investitionsüberlegungen erhalten (insbesondere Wärmepumpen, Holzfeuerungen in Form von Chips und Pellets und solarthermische Anlagen). Hält dieser Trend an, kann man mit Recht nicht nur von Energieeffizienz-Netzwerken, sondern auch von Klimaschutz-Netzwerken reden.

- Trends zu mehr Strombedarf je Produktionseinheit infolge von Prozesssubstitutionen wurden in einigen Fällen beobachtet (z.B. Trockenfertigung mit Verdopplung des Druckluftbedarfs, Reinraumfertigung zur Erhöhung der Qualität und der Lebensdauer). Daher lagen die erzielten Verminderungen der spezifischen $\mathrm{CO}_{2}$-Emissionen etwas unterhalb der erreichten Effizienzverbesserungen.

- Je intensiver sich die Energiebeauftragten mit dem Thema befassten, desto umfangreicher wurden ihre Kenntnisse und in der Regel auch ihre Akzeptanz bei der Geschäftsleitung. Sie veränderten betriebliche Regeln bei der Beschaffung bzw. im Einkauf und bei der wirtschaftlichen Bewertung von Investitionsmaßnahmen (interne Verzinsung statt oder in Ergänzung zur Amortisationszeit). Die Anwendung dieser Methodik führte nicht nur zu einer deutlichen Senkung der Energiekosten und Verbesserung des Klimaschutzes, sondern sie eröffnet den Unternehmen auch die Möglichkeit, durch aktiven Klimaschutz betriebswirtschaftliche Gewinne zu erzielen.

Abbau mangelnder Kenntnisse über die Potentiale und deren Nebeneffekte

- Der Kenntnisstand der Energieverantwortlichen der beteiligten Unternehmen war zu Beginn der Netzwerktreffen sehr unterschiedlich. Er verbesserte sich deutlich im Laufe der Treffen und des fortschreitenden Erfahrungsaustausches. Die Beschäftigung der Energiebeauftragten mit dem Thema führte nach wenigen Jahren zu Forderungen an die Anlagen- und Maschinenhersteller, ihre Produkte zu verbessern. Sie führte auch dazu, dass die Energieverantwortlichen dem Einkauf ihres Unternehmens technische Standards vorzugeben versuchten (z.B. Hocheffizienz-Elektromotoren für definierte Leistungen und Jahresnutzungsstunden).

- Lebenszykluskosten-Analysen werden von nur wenigen Betrieben zu Beginn des Netzwerkes gemacht und daher auch von den Herstellern in der Regel nicht angegeben, zum Teil auf Nachfragen auch gar nicht gewußt.

- Ebenso werden begleitende Nutzen bei Investitionen zur Energieeffizienz nur sehr selten beachtet (z.B. weniger Ausschuss, gleichmäßige Produktqualität, geringerer Lärm, bessere Arbeitsbedingungen, höhere Arbeits- oder Kapitalproduktivität).

- Der Erfolg der initiierten Netzwerke ist nicht zuletzt anhängig von einem engagierten Moderator und kompetenten beratenden Ingenieuren.

\section{Selbsthemmung der Wirtschaft bei den Effizienz-Innova-} tionen

Energieeffiziente Anlagen und Maschinen haben in vielen Fällen höhere Investitionen und entsprechende Kapitalkosten, aber geringere Energiekosten. Diese Substitution von Ressourcenkosten durch Kapitalkosten wird seitens der Hersteller energienutzender oder -wandelnder Anlagen und Maschinen nicht sachgerecht thematisiert.

- Die Hersteller von Anlagen und Maschinen konnten auf Befragen der Betriebe der Netzwerke zum Teil nicht den Energieverbrauch ihrer eigenen Produkte angeben. In einigen Fällen reagierten sie bei Anfragen für höher effiziente Anlagen und Maschinen mit einem „Nein, das machen wir nicht" oder mit einer Aussetzung der Gewährleistung. Die Gründe dieses innovationsfeindlichen Verhaltens sind vielfältig (Minimierung der Investitionen der Einkäufer ihrer Kunden, keine Segmentierung des Produktangebotes und damit verbunden eine komplizierte Produktionsplanung und kleinere Produktionsserien, Scheu vor weiteren Entwicklungskosten oder unbekannten Risiken, etc.). 
Tab. 1 Energieeffizienzgewinne und Verbesserung der spezifischen $\mathrm{CO}_{2}$-Emissionen (in \%) für vier Referenz-Netzwerke im Beobachtungszeitraum

\begin{tabular}{lllll}
\hline Netzwerk-Name & Zeitraum & Effizienz-Gewinn & $\mathrm{CO}_{2}$-Minderung & Methode \\
\hline Modell Hohenlohe & $2004-2008$ & $8,1 \%$ & $7,5 \%$ & top down \\
Ulm & $2004-2007$ & $5,9 \%$ & $24 \% 1$ & top down \\
- ohne Versorger & $2004-2008$ & $4,5 \%$ & $4 \%$ & top down \\
Mitteldeutschland $^{2}$ & $2005-2008$ & $8 \%$ & $6,6 \%$ & bottom up \\
Ost-Württemberg $^{\text {Ont }}$ & $2006-2008$ & $4 \%$ & $3,8 \%$ & top down \\
\hline
\end{tabular}

${ }^{1}$ Holz-Chips statt Gas einer KWK-Anlage

${ }^{2} 8$ von 13 Unternehmen

Quelle: Jochem et al. (2009)

- Die Hersteller von Anlagen und Maschinen bieten in fast allen Fällen ihre Effizienz-Produkte in den Kostenvergleichsrechnungen - sei es in ihren Angeboten oder ihren elektronischen Berechnungshilfen - nur das Ergebnis von Amortisationszeiten an, d.h. einem Risikomaß. Der zusätzliche Hinweis auf die Rentabilität der effizienteren Option in Form der internen Verzinsung oder des Barwertes ist extrem selten zu finden.

Damit ist die Selbsthemmung der Wirtschaft zu energieeffizienten Innovationen in sich geschlossen: Die Forderung nach niedrigen Investitionen (ohne Vorgabe von Effizienzstandards der Einkäufer) und die Investitionsentscheidung anhand von Amortisationszeiten seitens der Anwender führen zu vielen energieverschwenderischen Investitionen. Die Unternehmen der Netzwerke als Anwender sind aber auf eine größere Innovationsbereitschaft der HerstellerUnternehmen angewiesen.

Ermutigende Ergebnisse bei den beobachteten Energieeffizienz-Netzwerken

- Die beteiligten Unternehmen der Netzwerke gaben sich ein Effizienzziel von etwa 2\% pro Jahr (vgl. Tab. 1) was etwa doppelt so viel ist wie der Durchschnitt der Industriebetriebe in den letzten fünf Jahren. Dieses Ziel wurde auf der Netzwerkebene bei allen fünf beobachteten Netzwerken erreicht. Allerdings gab es erhebliche Abweichungen für einzelne Betriebe aus sehr unterschiedlichen Gründen (z.B. erhebliche/keine Neuinvestitionen, erhebliche Produktionsausweitung oder -einschränkung, große/geringe Unterstützung der Geschäftsleitung, vgl. Jochem et al. 2009).

- Die Ergebnisse zur $\mathrm{CO}_{2}$-Emissionsminderung lagen etwas unter $2 \%$ jährlich, weil der Trend zu relativ mehr Strombedarf in allen Netzwerken zu beobachten war und die spezifischen $\mathrm{CO}_{2}$-Emissionen des Strom höher liegen als die der Brennstoffe.

- Im Durchschnitt wurden pro Betrieb nach drei bis vier Jahren Energieeinsparungen von $100.000 €$ mit einem
Emissionsminderungserfolg von rd. $500 \mathrm{t} \mathrm{CO}_{2}$ pro Jahr erreicht.

- Sechs Unternehmen von 48 Unternehmen der fünf von den Autoren beobachteten Effizienz-Netzwerke erhielten in den letzten drei Jahren einen Energieeffizienz- oder Umweltpreis seitens der dena, der KfW oder anderer Institutionen.

- Die insgesamt 64 Betriebe der ersten fünf seitens der EnBW initiierten Netzwerke ergriffen insgesamt 318 Maßnahmen mit einer jährlichen Energiekosteneinsparung von rund $3 \mathrm{Mio}$. $€$ und jährlichen $\mathrm{CO}_{2}$-Emissionsminderungen von rd. $13.000 \mathrm{t}$ pro Jahr. Die EffizienzSteigerungen pro Jahr waren durchschnittlich 2,3\%.

- Eine Reihe der Netzwerk-Unternehmen betrachteten durch Impulse der Netzwerk-Arbeit ihre eigenen Produkte unter dem Gesichtspunkt der erhöhten Energieeffizienz (z.B. Ventilatoren, Getriebe) oder der Materialeffizienz (z.B. geringere Gewichte durch verbesserte Konstruktion oder andere Materialeigenschaften) und brachten hocheffiziente neue Produkte an den Markt.

- Die Anforderungen von Genauigkeit der Aussagen der verwendeten Monitoring-Verfahren zur jährlichen Energieeffizienzverbesserung eines Betriebes und der Verminderung der spezifischen $\mathrm{CO}_{2}$-Emissionen stehen im Widerspruch zu den Erwartungen vieler Energiebeauftragter in den Betrieben, diese Aussagen zu einem minimalen Aufwand der Datenbereitstellung zu erhalten.

Insgesamt kommen die Autoren zu dem Ergebnis, dass die lernenden Energieeffizienz-Netzwerke nicht nur ein sehr interessantes energie- und klimapolitisches Instrument für die Zukunft darstellen, das die Wirtschaft selbst in eigenem Interesse und in Eigenregie anwenden kann. Vielmehr ist es auch ein innovationspolitisches Instrument durch die Belebung der Nachfrage der Anwender nach Investitionsgütern mit hoher Energie- und Materialeffizienz. Je mehr Netzwerke entstehen und diese Nachfrage verstärken, desto größer ist der Kostensenkungseffekt der neuen Technologien durch Lern- und Skaleneffekte bei den Herstellern. 


\section{Ausblick}

Da das Potential der Energieeffizienz-Netzwerke in Deutschland, gemessen an den Schweizer Erfahrungen bei etwa 300 Netzwerken (ohne zusätzlichen finanziellen Anreiz) und bei rund 700 Netzwerken bei steuerlichen Erleichterungen liegt, wurde bald deutlich, dass viele Moderatoren und beratende Ingenieure für diese Aufgabe benötigt werden. Diese sollten möglichst nach einem Mindeststandard arbeiten, wie dies auch in der Schweiz durch die koordinierende Funktion der Energie-Agentur der Wirtschaft gewährleistet wird (Energie-Agentur der Wirtschaft 2009). Dies bedeutete zwei notwendige Schritte:

- Die Entwicklung eines Netzwerk-Managementsystems, das diese Funktion eines einheitlichen Mindestqualitätsstandards bei den einzelnen Arbeiten leistet. Diese Entwicklung wurde in den Jahren 2006-2009 durchgeführt und seitens der DBU und zweier Bundesländer finanziell gefördert.

- Der Aufbau einer bundesweit verteilten Anfangskompetenz von Initiatoren, Moderatoren und beratenden Ingenieuren, die als Ausgangspunkt für eine derartige Diffusion von Energieeffizienz-Netzwerken dienen kann. Diese Voraussetzung wird derzeit durch ein vom Bundesumweltministerium gefördertes 30-Pilotnetzwerke-Projekt erfüllt.

\subsection{Netzwerk-Managementsystem und dessen Verbreitung}

Um die Entwicklung des ${ }^{\circledR}$ LEEN-Managementsystems und die erforderlichen Schulungen für Moderatoren und beratende Ingenieure auf Basis einer neutralen Institution durchführen zu können, wurde im Jahre 2009 eine eigene Gesellschaft mit dem Namen LEEN GmbH von zwei Forschungseinrichtungen und einem Energieversorger gegründet. Für weitere Gesellschafter, z.B. die DIHK als Vertreter der Wirtschaft mit einer regionalen Struktur, oder die dena als bundesweite Energieeffizienz-Agentur, sind die drei Gründungsgesellschafter offen. Es ging zunächst einmal darum, schnell zu einer derartigen Gründung zu kommen und die Voraussetzungen zu schaffen, dass die für das 30-PilotNetzwerke-Projekt und für andere entstehende Netzwerke erforderlichen Moderatoren und beratenden Ingenieure ausgebildet und zertifiziert werden können.

Das Schulungskonzept für Moderatoren und beratende Ingenieure umfasst die Schulungs- und Prüfungsunterlagen, die Regeln der Voraussetzungen zur Teilnahme, das Prüfungskonzept und die Regeln der Zertifikatvergabe. Es hat einen gemeinsamen Teil, der für beide Zielgruppen wichtig ist und im wesentlichen das Zentral-Dokument des ${ }^{\circledR}$ LEENManagementsystems ist. Zum anderen werden Zielgruppenspezifische Materialien eingesetzt, die als spezielle Komponenten im LEEN-Managementsystem vorhanden sind. Die Materialien umfassen auch praktische Übungen.
Ein Großteil der Schulungsunterlagen wird jeweils einige Wochen vor dem Schulungstermin versandt, um die Teilnehmer über ein Selbststudium auf die weiterführende Schulung an dem eigentlichen Termin vorzubereiten und um ein zweimaliges Treffen zur Schulung zur vermeiden. Es werden auch Beispiele von Prüfungsunterlagen den Teilnehmern vorab zur Verfügung gestellt. Moderatoren mit unzureichender Moderationserfahrung hospitieren einige Male in Energieeffizienz-Treffen, um sich die nötige Praxis zu erarbeiten und das Zertifikat erhalten zu können.

Derzeit gibt es rund 45 zertifizierte Beratende Ingenieure und 45 zertifizierte Moderatoren in Deutschland.

\subsection{Das 30-Pilotnetzwerke-Projekt}

Ziele dieses zwischen dem Dezember 2008 und Juni 2013 laufenden Projektes sind,

- 30 Pilot-Netzwerke mit etwa 400 Betrieben bundesweit bei ihrer Initiierung und ihrem Betrieb der ersten drei bis vier Jahre zu unterstützen. Dabei werden ihre Kosten für Moderation, Initialberatung und Monitoring bis zu einem Drittel gefördert.

- Dadurch soll erreicht werden, dass die Kompetenz für Moderation, Initialberatung und Monitoring bundesweit verteilt wird und zugleich die Erfahrungen in den einzelnen Netzwerken allen Beteiligten zur Verfügung zu stellen.

- Außerdem sollen ca. 30 elektronische Investitionsberechnungshilfen für Energieeffizienz-Investitionen und Investitionsmöglichkeiten in erneuerbare Energien entwickelt werden. Nach Praxistest und Freigabe sollen diese Hilfen jedem beratenden Ingenieur gegen Zahlung einer Schutzgebühr zur Verfügung stehen. Hierdurch wird erreicht, dass die Investitionsberechnungen nach einem Mindestqualitätsmaßstab erfolgen und die Kosten der beratenden Ingenieure für derartige Dienstleistungen gesenkt werden können.

Durch diese Aktivitäten - den Erfahrungsaustausch bei den Netzwerktreffen sowie die EDV-basierten Investitionsberechnungshilfen für die beratenden Ingenieure werden die Transaktionskosten der Betriebe erheblich reduziert und zugleich die Leistungsfähigkeit und Produktivität der externen Beratung erhöht. Diese Verminderung der Transaktionskosten soll auch empirisch bei den Betrieben für Erst-Investoren und (nachahmende) Zweit-Investoren erhoben werden.

Damit zielt das Projekt auf die organisatorische Voraussetzung, bis zum Ende des kommenden Jahrzehnts das Potential von 400 bis 700 derartiger Netzwerke auszuschöpfen, die ihre $\mathrm{CO}_{2}$-Emissionen um etwa 10 Mio. t senken könnten.

Die Performance der 30 Netzwerke wird zwischen 2009 und 2013 laufend wissenschaftlich begleitet, es sollen Verbesserungen vorgenommen und Potentiale netzwerkübergreifender Synergien genutzt werden (z.B. gemeinsame 
Hotline, Austausch erfolgreicher Einzelmaßnahmen über eine website, Arbeitstreffen und Jahreskonferenzen). Diese Erfahrungen bei Moderatoren und den beratenden Ingenieuren sollen dann die Basis für eine schnelle Diffusion der Energieeffizienz- und Klimaschutz-Netzwerke in ganz Deutschland legen, und damit für eine schnelle Diffusion entsprechender Investitionen und Kenntnisse zur Energiekostensenkung in mittelständischen Betrieben in Deutschland.

Open Access Dieser Artikel unterliegt den Bedingungen der Creative Commons Attribution Noncommercial License. Dadurch sind die nichtkommerzielle Nutzung, Verteilung und Reproduktion erlaubt, sofern der/die Originalautor/en und die Quelle angegeben sind.

\section{Literatur}

Aijzen I (1991) The theory of planned behavior. Organ Behav Hum Decis Process 50:179

Bürki T (1999) Das Energie-Modell Schweiz als Erfahrungsfaktor für Schweizer Unternehmen. Bundesamt für Energie: Energie 2000, Ressort Industrie. Benglen

DeCanio S (1993) Barriers within firms to energy-efficient investments. Energy Policy 9:906

DeCanio SJ (1998) The efficiency products: bureaucratic and organisational barriers to profitable energy saving investments. Energy Policy 26:441

DeGroot HLF et al (2001) Energy savings by firms: decision-making, barriers and policies. Energy Econ 23:717

Dosi G (1988) The nature of the innovative process. In: Dosi G et al (Hrgs) Technical change and economic theory. Springer, Berlin, S 221

Energie-Agentur der Wirtschaft (EnAW) (2009) Jahresbericht 2008. Energie Schweiz, Zürich

InterSEE (1998) Interdisciplinary analysis of successful implementation of energy efficiency in industry, commerce and service. Wuppertal Institut für Klima Umwelt Energie, AKF-Institute for Local Government Studies, Energieverwertungsagentur, Fraunhofer Institut für Systemanalyse und Innovationsforschung, Institut für Psychologie der Universität Kiel, Amstein\&Walthert, Bush Energie (Hrsg.), Wuppertal, Kopenhagen, Wien, Karlsruhe, Kiel
Jansen D (1999) Einführung in die Netzwerkanalyse. Grundlagen, Methoden, Anwendungen. Leske \& Budrich, Opladen

Jochem E, Gruber E (2007) Local learning networks on energy efficiency in industry-successful initiative in Germany. Appl Energy $84: 806-816$

Jochem E et al (2000) Society, behaviour, and climate change. advances in global change research. Kluwer Academic, Dordrecht

Jochem E, Gruber E, Weissenbach K, Westdickenberg J, Feihl M, Ott V (2006) Modellvorhaben Energieeffizienz-Initiative Region Hohenlohe zur Reduzierung der $\mathrm{CO}_{2}$-Emissionen 2002-2006. Schlussbericht für das Umweltministerium Baden-Württemberg. Karlsruhe/Waldenburg

Jochem E, Ott V, Weissenbach K (2007) Lernende Netzwerke - einer der Schlüssel zur schnellen Energiekostensenkung. Energiewirtschaftliche Tagesfragen 57:8-11

Jochem E, Bauer J, Weissenbach K, Bradke H, Köwener D, Ott V (2009) Umweltkommunikation und Energieeffizienz in KMU: Moderierte EnergieEffizienz-Tische in Unternehmensnetzwerken reduzieren $\mathrm{CO}_{2}$. Schlußbericht für die DBU, Karlsruhe/Waldenburg

Konersmann L (2002) Energieeffizienz in der Wirtschaftthe - Evaluation des EnergieModell Schweiz und Konzeption eines MultiAgenten-Modells. Master Thesis. ETH Zurich

Kristof K et al (1999) Evaluation der Wirkung des Energie-Modells Schweiz auf die Umsetzung von Maßnahmen zur Steigerung der Energieeffizienz in der Industrie und seiner strategischen energiepolitischen Bedeutung. Bundesamt für Energie, Bern

Loistl M, Weissenbach K (2007) Energieeffizienztische - lernende Netzwerke. Brennst. Wärme Kraft 59(9):1-3

Ostertag K (2003) No-regret potentials in energy conservation. An analysis of their relevance, size and determinants. Physica Verlag, Heidelberg

Schmid Chr (2003) Energieeffizienz in Unternehmen. Eine wissensbasierte Analyse von Einflussfaktoren und Instrumenten. VDF, Zürich

Scott J (2000) Social network analysis. A handbook, 2. Aufl. Sage, London

Sorrell S et al (2000) Reducing barriers to energy efficiency in private and public organisations. Final report. University of Sussex, Brighton

Stern PC (1992) What psychology knows about energy conservation. Am Psychol 47:1224-1232

Wagner Th (2008) Am besten im Netzwerk - Höhere Energieeffizienz im Mittelstand. BWK 10(1-2):8-9 